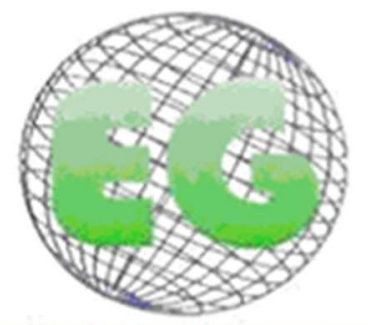

ISSN 1695-6141 $\mathbf{N}^{\circ} 63$

\title{
ORIGINALES
}

\section{Confiabilidad del instrumento Seguridad del Paciente en Administración de Medicamentos en Pediatría- Versión española} Confiabilidade do instrumento Segurança do Paciente na Administração de Medicamentos na Pediatria- Versão española

Reliability of the patient safety instrument in drug administration in pediatrics - Spanish version

\author{
Lilia Jannet Saldarriaga Sandoval ${ }^{1}$ \\ Francisca Elisângela Teixeira Lima² \\ Paulo César de Almeida ${ }^{3}$ \\ Lorena Pinheiro Barbosa ${ }^{2}$ \\ Sabrina de Souza Gurge ${ }^{2}$ \\ Lívia Maia Pascoal ${ }^{4}$ \\ 1 Universidad Nacional de Tumbes. Tumbes Perú. liyasa45@hotmail.com \\ 2 Universidad Federal de Ceará. Fortaleza-Ceará, Brasil. \\ 3 Universidad Estadual de Ceará. Fortaleza-Ceará, Brasil \\ ${ }^{4}$ Universidad Federal do Maranhão, São Luís -Brasil.
}

https://doi.org/10.6018/eglobal.442261

Recibido: 4/09/2020

Aceptado: 10/01/2021

\section{RESUMEN:}

Objetivo: Evaluar las propiedades psicométricas en términos de confiabilidad del instrumento Seguridad del Paciente en la Administración de Medicamentos en Pediatria, versión española.

Método: Estudio metodológico de evaluación de las propiedades psicométricas realizado con 25 enfermeras de las unidades pediátricas de un hospital peruano. Para evaluación de la homogeneidad (alfa de Cronbach) las enfermeras autocompletaron el instrumento de Seguridad del Paciente en la Administración de Medicamentos en Pediatria version española, compuesto por 9 dominios y 26 ítems; y para estabilidad (Test-retest de Wilcoxon) autocompletaron nuevamente el intrumento después de 30 dias de la primera recolección, para comparación de ambas medidas.

Resultados: En la confiabilidad, el alfa de Cronbach, vario de 0,792 a 0,821, considerado un parámetro aceptable, presentando alta consistencia interna, manteniéndose los 26 ítems, en la versión final. En la estabilidad del instrumento, los dominios presentaron, en el test, media de 68,0 $\pm 25,5$ hasta 99,5 \pm 2,5; y en el retest $86,0 \pm 14,8$ até $96 \pm 11,8$. Se evidenciaron seis dominios sin diferencia significativa $(p>0,05)$ entre test y retest.

Conclusión: El instrumento presentó propiedades psicométricas que comprueban su confiabilidad, proporcionando subsídios para la práctica de enfermería más segura y permitiendo estandarización del cuidado en la administración de medicamentos.

Palabras clave: Seguridad del Paciente; Utilización de Medicamentos; Enfermería Pediátrica; Psicometría; Reproducibilidad de los Resultados; Pediatría. 


\title{
RESUMO:
}

Objetivo: Avaliar as propriedades psicométricas em termos de confiabilidade do instrumento Segurança do Paciente na Administração de Medicamentos na Pediatria, versão espanhol.

Método: Estudo metodológico realizado com 25 enfermeiras das unidades pediátricas de um hospital peruano. Para avaliação da homogeneidade (alfa de Cronbach) as enfermeiras autopreencheram o SPAMP-vE, composto por nove domínios e 26 itens; e para estabilidade (Teste-reteste de Wilcoxon) autopreencheram novamente o intrumento após 30 dias da primeira coleta, para comparação de ambas medidas.

Resultados: Na confiabilidade, o alfa de Cronbach, variou de 0,792 a 0,821 , considerado um parâmetro aceitável, apresentando alta consistência interna, mantendo-se os 26 itens, na versão final. Na estabilidade do instrumento, os domínios apresentaram, no teste, média de $68,0 \pm 25,5$ até $99,5 \pm$ 2,5 ; e no reteste $86,0 \pm 14,8$ até $96 \pm 11,8$. Evidenciaram-se seis domínios sem diferença significativa $(p>0,05)$ entre teste e reteste.

Conclusão: Instrumento apresentou propriedades psicométricas que comprovam sua confiabilidade, fornecendo subsídios para prática de enfermagem mais segura e permitindo padronização do cuidado na administração de medicamentos.

Palavras-chave: Segurança do Paciente; Uso de Medicamentos; Enfermagem Pediátrica; Psicometria; Reprodutibilidade dos Testes; Pediatria.

\begin{abstract}
:
Objective: To evaluate the psychometric properties in terms of reliability of the instrument Patient Safety in the Administration of Medicines in Pediatrics, Spanish version.

Method: Methodological study of evaluation of the psychometric properties carried out with 25 nurses from the pediatric units of a Peruvian hospital. For the evaluation of homogeneity (Cronbach's alpha), the nurses completed the instrument for Patient Safety in the Administration of Medications in Pediatrics Spanish version, composed of nine domains and 26 items; and for stability (Wilcoxon test-retest), they autocompleted the instrument again 30 days after the first collection, for comparison of both measurements.

Results: In reliability, Cronbach's alpha varied from 0.792 to 0.821 , considered an acceptable parameter, presenting high internal consistency, maintaining the 26 items, in the final version. In the stability of the instrument, the domains presented, in the test, a mean of $68.0+25.5$ to $99.5+2.5$; and in the retest $86.0+14.8$ I tied $96+11.8$. Six domains were found without significant difference $(p>0.05)$ between test and retest.

Conclusion: The instrument presented psychometric properties that prove its reliability, providing subsidies for safer nursing practice and allowing standardization of care in the administration of medications.
\end{abstract}

Descriptors: Patient Safety; Drug Utilization; Pediatric Nursing; Psychometrics; Reproducibility of Results; Pediatrics.

\section{INTRODUCCIÓN}

El sistema de medicación consta de varios procesos, como prescripción, dispensación, preparación, administración y acompañamiento del paciente para monitoreo de la acción o reacción al medicamento(1). Es competencia de todos los miembros involucrados en la prestación del cuidado en salud, siendo el profesional de enfermería el responsable de la preparación y administración de medicamentos, la cual es una de las actividades mas complejas debido a los errores o casi errores que son más frecuentes en niños hospitalizados que en adultos ${ }^{(2)}$, lo que puede causar daños gravísimos y hasta muerte, además de generar altos costos anualmente en todo el mundo(3).

Dentro de los errores de medicación existentes en pediatría, la mayoría es de administración, siendo la dosis incorrecta el tipo de error más frecuente, seguida por la omisión de medicación y por la medicación administrada en el momento 
incorrecto(4). Así, se observa una amplia variedad de posíbles errores involucrados en cada una de las etapas de la administración de medicamentos, ligada directamente al cuidado de enfermería, lo que va en contra del concepto de seguridad del paciente que trata sobre la reducción, a un mínimo aceptable, del riesgo de daño innecesario asociado al cuidado de salud(5).

Por lo tanto, es necesaria la promoción de la seguridad del paciente en administración de medicamentos. Asi, en Brasil fue desarrollado y validado el instrumento Seguridad del Paciente en la Administración de Medicamentos en Pediatría (SPAMP), con Índice de Validez de contenido (IVC) de 0,938 y alfa de Cronbach de $0,851^{(6)}$. Es una herramienta para el diagnóstico de la seguridad del paciente en el proceso de administración de medicamentos capaz de evaluar las acciones de la seguridad del paciente durante la administración de medicamentos en niños hospitalizados en pediatría, pues contempla el protocolo de seguridad en la prescripción, uso y administración de medicamentos ${ }^{(7)}$.

El instrumento SPAMP es el único construido en versión portuguesa que ha sido traducido, adaptado, de acuerdo con Beaton ${ }^{(8)}$, y validado para el idioma español, el Instrumento Seguridad del Paciente en la Administración de Medicamentos en Pediatria (SPAMP-vE) en el contexto de Perú(9), el cual alcanzó un Coeficiente de Validez de Contenido (CVC) de 0,97 para pertinencia práctica, 0,97 para claridad de lenguaje y 0,96 para relevancia teórica. Está compuesto por 26 ítems distribuidos en nueve dominios relacionados con la seguridad en la administración de medicamentos en niños ${ }^{(7)}$. Para ser usado en el Perú con los profesionales que preparan y administran medicamentos, el instrumento debe demostrar concordancia y consistencia interna.

El estudio se justifica porque el instrumento SPAMP-vE es una herramienta apropiada para evaluar acciones de la promoción de la seguridad del paciente en la administración de medicamentos ejecutadas por los profesionales de enfermería en las unidades de internación. De esa forma, para la aplicación del instrumento traducido y adaptado junto a las enfermeras cuidadoras de los niños, se hace necesario que sus propiedades psicométricas sean evaluadas, como en el caso la confiabilidad, considerando la estabilidad y homogeneidad del instrumento.

La confiabilidad de un instrumento permite que se conozca el grado en que el mismo reproduzca de forma consistente los resultados aplicados en diferentes ocasiones además de representar una de las principales propiedades de medida, la cual precisa ser evaluada cuando se desarrolla una nueva medida, y ofrece informaciones sobre la necesidad de mejora de un instrumento ya existente ${ }^{(10)}$.

De ese modo, se tuvo como objetivo evaluar las propiedades psicométricas en el aspecto de confiabilidad del instrumento Seguridad del Paciente en la Administración de Medicamentos en Pediatria, versión español.

\section{MÉTODO}

Estudio metodológico de evaluación de las propiedades psicométricas del instrumento SPAMP-vE realizado con las enfermeras responsables por la preparación, administración de medicamentos y monitorización de los niños de un hospital en Perú 
durante el período de abril a mayo 2018. Siguiendo los preceptos éticos vigentes en cada país, el estudio obtuvo aprobación en el Brasil por el Comité de Ética en investigación de la Universidad Federal del Ceará (dictamen favorable $n^{\circ} 2.583 .089$ ) y en el Perú por el Comité de Ética del referido hospital (código 0211-052-17) y el consentimiento informado de los participantes en el estudio.

El universo del estudio estuvo conformado por 220 enfermeras que laboran en el hospital de las cuales se eligieron solo a las enfermeras que laboran en pediatría, dado que las acciones de la administración de medicamentos a los niños hospitalizados en pediatría se evalúan con el instrumento SPAMP-vE. La muestra estuvo compuesta por el total de 25 enfermeras de pediatría que atendieron los siguientes criterios de inclusión: actuar en el proceso de administración de medicamentos de niños y adolescentes hospitalizados y tener vínculo profesional en el hospital de por lo menos seis meses. Los criterios de exclusión fueron: encontrarse de vacaciones, licencia o apartado de sus actividades en el período de recolección de datos; ser enfermero cuyo sector de origen no fuese aquel escogido para ser objeto de investigación.

El instrumento SPAMP-vE, traducido y adaptado culturalmente, posee 26 ítems, distribuidos en nueve domínios, los cuales son: 1- Paciente correcto; 2- Medicamento correcto; 3- Vía correcta; 4- Hora correcta; 5- Dosis correcta; 6- Registro correcta; 7Orientación correcta; 8- Forma correcta; y 9- Respuesta correcta. Se considera como una escala Likert, en la que cada elemento consta de cinco puntos, que varían, de 1 a 5 , siendo 1-nunca, 2-casi nunca, 3-a veces, 4-casi siempre y 5-siempre, con puntuación entre 26 a 130 puntos.

Para recolección de datos, las enfermeras autocompletaron el instrumento SPAMP-vE en el día de su turno, marcando en los ítems la puntuación correspondiente a su práctica en el proceso de administración de medicamentos, conforme a lo recomendado por la autora del SPAMP versión en portugués(6). Después de 30 días, se realizó la aplicación del mismo instrumento a las enfermeras que autocompletaron nuevamente el instrumento SPAMP-vE para comparación de ambas medidas. El tiempo medio para que la enfermera autocompletara el instrumento fue de 10 minutos, variando entre 5 y 15 minutos.

Para homogeneidad, se usó el alfa de Cronbach para evaluación de la pertinencia práctica, claridad de lenguaje y relevancia teórica. Se trata de un coeficiente que produce valores entre 0 y 1 , o sea, entre 0 y $100 \%$, siendo considerado satisfactorio para el presente estudio los valores mayores de $70 \%(11)$. Y para estabilidad, se utilizó el test-retest con el objeto de analizar la correlación entre los resultados de las dos aplicaciones, por medio del test de Wilcoxon ${ }^{(12)}$, considerando significancia estatística $p<0,05$.

\section{RESULTADOS}

Las 25 enfermeras que participaron del estudio son todas de sexo femenino, el rango de edad varió de 27 a 41 años con un promedio de $33.3(+3.8)$ años y un predominio del grupo de edad de $27-31$ años (44.0\%), en cuanto a su formación profesional, el $84.0 \%$ tenía otra especialidad y no es pediatría, el $48.0 \%$ de los profesionales tenía más de tres a cinco años de capacitación y el $48.0 \%$ informó lo mismo de seis a diez 
años de experiencia profesional, señalando que el $72.0 \%$ de ellos tienen entre dos y cuatro años de experiencia en pediatría, el $40.0 \%$ de las enfermeras mencionó una carga de trabajo semanal promedio de 37 horas y $100.0 \%$, el $52.0 \%$ tiene un contrato de trabajo.

En el caso de la homogeneidad y la confiabilidad del instrumento SPAMP-vE en los 26 ítems, el alfa de Cronbach vario de 0,792 a 0,821 , el que demostró una alta consistencia interna del instrumento, con una media de 0,812 , ratificando el mantenimiento de los 26 ítems en la versión final (Tabla 1).

Tabla 1 - Valores de alfa de Cronbach en ausencia de alguno de los ítems del instrumento. Lambayeque, Perú, 2019 (N=26 ítems)

\begin{tabular}{|c|c|}
\hline Ítem & Alfa de Cronbach si el ítem fuera excluido \\
\hline Ítem 1 & 0,817 \\
\hline Ítem 2 & 0,823 \\
\hline Ítem 3 & 0,820 \\
\hline Ítem 4 & 0,798 \\
\hline Ítem 5 & 0,792 \\
\hline Ítem 6 & 0,818 \\
\hline Ítem 7 & 0,822 \\
\hline Ítem 8 & 0,814 \\
\hline Ítem 9 & 0,819 \\
\hline Ítem 10 & 0,820 \\
\hline Ítem 11 & 0,808 \\
\hline Ítem 12 & 0,816 \\
\hline Ítem 13 & 0,824 \\
\hline Ítem 14 & 0,817 \\
\hline Ítem 15 & 0,820 \\
\hline Ítem 16 & 0,809 \\
\hline Ítem 17 & 0,798 \\
\hline Ítem 18 & 0,808 \\
\hline Ítem 19 & 0,800 \\
\hline Ítem 20 & 0,804 \\
\hline Ítem 21 & 0,821 \\
\hline Ítem 22 & 0,814 \\
\hline Ítem 23 & 0,806 \\
\hline Ítem 24 & 0,813 \\
\hline Ítem 25 & 0,805 \\
\hline Ítem 26 & 0,821 \\
\hline
\end{tabular}

Fuente; datos de la investigación

En el test, los dominios del instrumento SPAMP-vE presentaron media y desviación estándar de 68,0 $\pm 25,5$ hasta $99,5 \pm 2,5$; y en el retest de 86,0 $\pm 14,8$ hasta $96 \pm$ 11,8 , respectivamente. En la evaluación de la estabilidad, de los nüeve dominios del instrumento SPAMP-vE, seis no presentaron diferencia significativa $(p>0,05)$ entre el test y el retest, segun el test de Wilcoxon. Estos resultados demostraron que la asociación entre las dos aplicaciones fue satisfactoria, a pesar de tres dominios 
(Paciente Correcto, Dosis correcta y Respuesta correcta) hayan presentado diferencia estadisticamente significante (Tabla 2).

Tabla 2 - Comparación de las medias y desviación estándar de los dominios del instrumento SPAMP-vE en el test-retest. Lambayeque, Perú, $2019(\mathrm{~N}=25)$

\begin{tabular}{|c|c|c|c|c|c|}
\hline \multirow[t]{2}{*}{ Domínios } & \multicolumn{2}{|c|}{ Test } & \multicolumn{2}{|c|}{ Retest } & \multirow[b]{2}{*}{$p^{\dagger}$} \\
\hline & Media $\pm D P^{*}$ & Mediana & Media $\pm D P^{*}$ & Mediana & \\
\hline Paciente correcto & $68,0+25,5$ & 50,0 & $96,0+11,8$ & 100,0 & $<0,0001$ \\
\hline $\begin{array}{l}\text { Medicamento } \\
\text { correcto }\end{array}$ & $91,0 \pm 9,9$ & 93,7 & $86,0 \pm 14,8$ & 93,7 & 0,172 \\
\hline Vía correcta & $93,2 \pm 10,0$ & 100,0 & $92,7 \pm 7,6$ & 93,7 & 0,550 \\
\hline Hora correcta & $91,3 \pm 10,3$ & 91,6 & $93,0 \pm 9,2$ & 100,0 & 0,524 \\
\hline Dosis correcta & $87,6 \pm 10,5$ & 85,0 & $93,8 \pm 7,1$ & 95,0 & $<0,039$ \\
\hline Registro correcto & $81,2 \pm 17,1$ & 81,2 & $88,5 \pm 15,1$ & 100,0 & 0,055 \\
\hline $\begin{array}{l}\text { Orientación } \\
\text { correcta }\end{array}$ & $84,5 \pm 18,8$ & 100,0 & $91,0 \pm 12,2$ & 100,0 & 0,149 \\
\hline Forma correcta & $96,0 \pm 9,3$ & 100,0 & $93,0 \pm 11,4$ & 100,0 & 0,317 \\
\hline Respuesta correcta & $99,5 \pm 2,5$ & 100,0 & $95,5 \pm 7,1$ & 100,0 & $<0,011$ \\
\hline Instrumento total & $88,7 \pm 5,8$ & 90,4 & $91,5 \pm 7,0$ & 93,3 & 0,063 \\
\hline
\end{tabular}

${ }^{*} \mathrm{DE}$ : Desviación estandar; ${ }^{\dagger} \mathrm{p}$ : Test de Wilcoxon.

Esa etapa posibilitó la elaboración de la versión final del instrumento de seguridad del paciente en la administración de medicamentos en pediatria (SPAMP-vE), con 26 ítems distribuídos en nueve domínios (Cuadro 1).

Cuadro 1 - Versión final traducida para el español del instrumento Seguridad del Paciente en la Administración de Medicamentos (SPAM-vE). Lambayeque, Perú, 2019. 


\begin{tabular}{|c|c|c|c|c|c|c|c|}
\hline \multirow{2}{*}{\multicolumn{2}{|c|}{...气 }} & \multirow[t]{2}{*}{ ADMINISTRACION DE MEDICAMENTOS } & 宸 & 宸 & 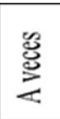 & 氮 育 & 鵕 \\
\hline & & & $\mathbf{1}$ & 2 & 3 & 4 & 5 \\
\hline 宸 & 题 & $\begin{array}{l}\text { 1. Utiliza al menos dos identificadores (nombre completo del } \\
\text { niño, fecha de nacimiento o número de historia clinica) para } \\
\text { identificar al niño antes de administrar medicamento. }\end{array}$ & & & & & \\
\hline \multirow{4}{*}{\multicolumn{2}{|c|}{ 电 }} & $\begin{array}{l}\text { 2. Confirma el nombre del medicamento con la prescripción } \\
\text { médica antes de administrarlo al niño. }\end{array}$ & & & & & \\
\hline & & $\begin{array}{l}\text { 3. Lleva a la cama solo los medicamentos prescritos a un único } \\
\text { niño. }\end{array}$ & & & & & \\
\hline & & $\begin{array}{l}\text { 4. Administra el medicamento por orden verbal solamente en } \\
\text { caso de emergencia. }\end{array}$ & & & & & \\
\hline & & $\begin{array}{l}\text { 5. Confirma si el niño no es alérgico al medicamento prescrito, } \\
\text { identificándolo de forma diferenciada con pulsera y aviso en la } \\
\text { historia clinica, alertando a todo el equipo. }\end{array}$ & & & & & \\
\hline \multirow{4}{*}{ 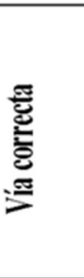 } & & \begin{tabular}{|llllllll}
$\begin{array}{l}\text { Identifica la via de } \\
\text { medicamento }\end{array}$ & administración prescrita para el \\
\end{tabular} & & & & & \\
\hline & & $\begin{array}{l}\text { 7.Verifica si la vía prescrita es la técnica recomendada para } \\
\text { administrar el medicamento }\end{array}$ & & & & & \\
\hline & & $\begin{array}{l}\text { 8. Lava las manos antes de la preparación y administración de } \\
\text { medicamentos. }\end{array}$ & & & & & \\
\hline & & $\begin{array}{l}\text { 9. Utiliza materiales y técnicas asépticas para administrar } \\
\text { medicamentos según las diferentes vias de administración. }\end{array}$ & & & & & \\
\hline \multirow{3}{*}{\multicolumn{2}{|c|}{ 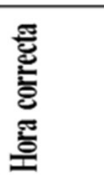 }} & $\begin{array}{lllll}\text { 10. Prepara el medicamento inmediatamente antes de su } \\
\text { administración. }\end{array}$ & & & & & \\
\hline & & 11. Administra el medicamento en la hora correcta. & & & & & \\
\hline & & $\begin{array}{l}\text { 12. Adecua los horarios de administración de los medicamentos a } \\
\text { la rutina ya establecida antes de su hospitalización. }\end{array}$ & & & & & \\
\hline \multirow{5}{*}{\multicolumn{2}{|c|}{ 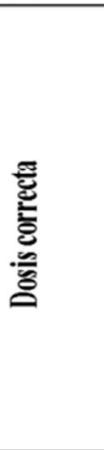 }} & $\begin{array}{l}\text { 13. Confirma atentamente la dosis según la prescripción médica } \\
\text { para el medicamento. }\end{array}$ & & & & & \\
\hline & & $\begin{array}{l}\text { 14. Confirma la velocidad del } \\
\text { funcionamiento de bombas deo, la programación y } \\
\text { prescripción médica. }\end{array}$ & & & & & \\
\hline & & $\begin{array}{l}\text { 15. Realiza doble chequeo por dos profesionales, de los cálculos } \\
\text { de dilución y administración de medicamentos potencialmente } \\
\text { peligrosos o medicamentos de alta vigilancia. }\end{array}$ & & & & & \\
\hline & & $\begin{array}{l}\text { 16. Utiliza instrumentos de medida estándar en la preparación de } \\
\text { medicamentos para medir la dosis exacta (ej: jeringas } \\
\text { milimetradas, vasos dosificados) }\end{array}$ & & & & & \\
\hline & & $\begin{array}{l}\text { 17. Devuelve a la farmacia las sobras de medicamentos no } \\
\text { administrados. }\end{array}$ & & & & & \\
\hline \multirow{4}{*}{\multicolumn{2}{|c|}{ 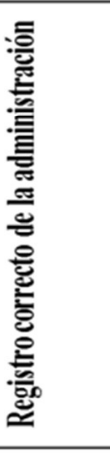 }} & $\begin{array}{l}\text { 18. Registra en el Kardex el horario de la administración y en la } \\
\text { historia clínica ocurrencias del medicamento inmediatamente } \\
\text { después de administrar cada dosis. }\end{array}$ & & & & & \\
\hline & & $\begin{array}{l}\text { 19. Notifica en la Ficha de Registro y reporte de incidentes, } \\
\text { reacciones adversa y eventos adversos a la oficina de Gestión de } \\
\text { Calidad. }\end{array}$ & & & & & \\
\hline & & $\begin{array}{l}\text { 20. Mantiene registro adecuado de medicamentos preparados que } \\
\text { serán almacenados (con fecha y horario de la manipulación, } \\
\text { concentración del medicamento, nombre del responsable por la } \\
\text { preparación y la validez). }\end{array}$ & & & & & \\
\hline & & $\begin{array}{l}\text { 21. Monitorea la temperatura de la refrigeradora de } \\
\text { acondicionamiento de los medicamentos registrando los valores } \\
\text { diariamente. }\end{array}$ & & & & & \\
\hline \multirow{2}{*}{\multicolumn{2}{|c|}{ 凑 }} & $\begin{array}{l}\text { 22. Aclara dudas sobre inelegibilidad de la prescripción, } \\
\text { indicación del medicamento e posologia antes de administrar el } \\
\text { medicamento. }\end{array}$ & & & & & \\
\hline & & $\begin{array}{l}\text { 23. Orienta al niño y al acompañante sobre el medicamento } \\
\text { administrado y la justificación, de la indicación, la frecuencia } \\
\text { con que será administrado y los efectos esperados. }\end{array}$ & & & & & \\
\hline & 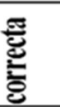 & $\begin{array}{l}\text { 24. Verifica si el medicamento a ser administrado posee la } \\
\text { presentación farmacéutica compatible con la via de } \\
\text { administración de la prescripción médica. }\end{array}$ & & & & & \\
\hline \multirow{2}{*}{\multicolumn{2}{|c|}{ 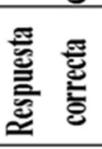 }} & $\begin{array}{l}\text { 25. Evalúa al niño para identificar cuando sea posible, si el } \\
\text { medicamento tuvo el efecto deseado. }\end{array}$ & & & & & \\
\hline & & $\begin{array}{l}\text { 26. Informa al médico que prescribió todos los efectos diferentes } \\
\text { de lo esperado (en intensidad y forma) para el medicamento. }\end{array}$ & & & & & \\
\hline
\end{tabular}




\section{DISCUSIÓN}

El instrumento SPAMP-vE proporcionó propiedades psicométricas semejantes al instrumento SPAMP en la versión en portugués. El alfa de Cronbach en el Instrumento SPAMP, versión en portugués, vario de 0,841 a $0,855^{(6)}$, manteniéndose también en el parámetro aceptable ${ }^{(11)}$. Resultados similares fueron presentados en otro instrumento de prácticas seguras para administración de medicamentos, constituido por 54 ítems divididos en 10 áreas, cuyo nivel de concordancia vario de 90 a $100 \%$ y alfa de Cronbach de $0,94^{(13)}$.

Otro estudio que utilizó la versión en portugués del Medical Office Survey on Patient Safety Culture para evaluación de la cultura de seguridad del paciente en la Atención Primaria a la salud en Brasil presento alfa de Cronbach de 0,95(14).

Al comparar los nueve dominios de la sección, tres (Paciente Correcto, Dosis correcta y Respuesta correcta) presentaron diferencia significativa $(p>0,05)$ entre el test y el retest. Estos dominios también presentaron diferencia significativa en la versión original en portugués( ${ }^{(6)}$. Así, el test-retest permitió prever que el comportamiento de las enfermeras puede ser alterado a lo largo de diferentes períodos de tiempo ${ }^{(15)}$, ya que es posible que pueda haber habido una sensibilización en cuanto a la administración segura de medicamentos después de autocompletar el instrumento, lo que llevó a la intención de mejorar el comportamiento ${ }^{(16)}$.

Sin embargo, a pesar de la variación de respuestas entre tres dominios en el testretest, la associación entre las dos aplicaciones fue satisfactoria, demostrando estabilidad para uso en comparación de medidas individuales ${ }^{(17)}$. De esa forma, el test-retest presentó valores que confirmaron la confiabilidad del instrumento.

Finalmente, la confiabilidad tanto en la homogeneidad como la estabilidad, consideradas las principales propiedades de medida del instrumento SPAMP-vE, evidenció que el mismo tiene la capacidad de reproducirse de forma consistente, en el tiempo y en el espacio, de modo que asegura la calidad de los resultados para ser aplicado en otros estudios.

Esta versión en español puede ser considerada como la primera versión confiable de español de la versión original, ya que, por medio del alfa de Cronbach, presentó alta confiabilidad, manteniéndose en el parámetro aceptable $^{(11)}$.

Además, la aplicación clínica de este instrumento comprendió las acciones de seguridad en la administración de medicamentos en pediatria por medio de una nueva herramienta válida y confiable en las poblaciones españolas de enfermeras en relación a la ejecución de los nueve correctos de la preparación y administración de la medicación.

De esa forma, mediante el creciente número de estudios realizados por enfermeras con la intención de traducir, adaptar y validar instrumentos, la adopción de técnicas y métodos con propiedades psicométricas satisfactorias es necesario, además de evaluar confiabilidad y validad para asegurar la calidad y rigor metodológico de la investigación ${ }^{(18)}$. Aunque, otros modelos de validación precisan ser investigados en la aplicación del instrumento en la práctica clínica en Enfermería para subsidiar el 
desarrollo de la escala y su incorporación en actividades rutinarias para administración de medicamentos en pediatria, y obtener una práctica más segura.

Como limitación, se destaca el hecho de que el instrumento ha sido validado en un único hospital y con enfermeras en pediatria. De acuerdo con la literatura, la confiabilidad de un instrumento no es una propiedad de medida fija, por lo tanto, la confiabilidad de un mismo instrumento puede variar dependiendo del contexto de la población que ha sido evaluada(19).

Se considera que la evaluación de las propiedades psicométricas del instrumento SPAMP-vE puede contribuir al avance del conocimiento científico, a medida que disponibiliza un instrumento válido y confiable, el cual puede ser aplicado en la práctica clínica del equipo de enfermería, con objeto de la calificación de la asistencia prestada y a la promoción de la seguridad en la administración de medicamentos del paciente pediátrico hospitalizado.

\section{CONCLUSIÓN}

El instrumento de Seguridad del paciente en la administración de medicamentos en la Pediatria versión en español (SPAMP-vE) es considerado confiable, una vez que la confiabilidad presento alta consistencia interna, con versión final constituida por 26 ítems, distribuidos en nueve dominios.

El estudio disponibiliza el instrumento SPAMP-vE para evaluar la seguridad del paciente en la administración de medicamentos en pediatria, ya que instrumentos válidos y confiables pueden estandarizar el cuidado y la realización adecuada de procedimientos específicos de Enfermería para promoción de la seguridad del paciente.

\section{Agradecimiento}

Universidad Federal de Ceará.Hospital Regional Lambayeque, enfermeras, estudiantes y profesoras del Programa de Posgraduación de Enfermería.

\section{REFERENCIAS}

1. Harada MJCS, Chanes DC, Kusahara DM, Pedreira MLG. Safety in medication administration in pediatrics. Acta paul. enferm. 2012; 25(4): 639-42. doi: 10.1590/S0103-21002012000400025.

2. Drovandi A, Robertson K, Tucker M, Robinson N, Perks S, Kairuz T. A systematic review of clinical pharmacist interventions in paediatric hospital patients. European Journal of Pediatrics. 2018;177(8):1139-48. doi: 10.1007/s00431-018-3187-x

3. Choi I, Lee SM, Flynn L, Kim CM, Lee S, Kim NK, et al. Incidence and treatment costs attributable to medication errors in hospitalized patients. Res Social Adm Pharm. 2016;12(3):428-37. doi: 10.1016/j.sapharm.2015.08.006

4. Truter A, Schellack N, Meyer JC. Identifying medication errors in the neonatal intensive care unit and paediatric wards using a medication error checklist at a tertiary academic hospital in Gauteng, South Africa. S. Afr. J. Child Health. 2017; 11(1):5-10. doi: 10.7196/SAJCH.2017.v11i1.1101. 
5. World Health Organization. Conceptual Framework for the International Classification for Patient Safety: Final Technical Report [Internet]. Geneva: WHO; 2009 [cited $2020 \quad$ Marc 08]. Available from: http://www.who.int/patientsafety/taxonomy/icps_full_report.pdf

6. Araújo PR, Lima FET, Ferreira MKM, Oliveira SKP, C̄arvalho REFL, Almeida PC. Medication administration safety assessment tool: Construction and validation. Rev. Bras. Enferm. 2019; 72(2):329-36. doi: 10.1590/0034-7167-2018-0340.

7. Ministério da Saúde (BR). Anvisa. Fiocruz. Fleming. Anexo 03: Protocolo de segurança na prescrição, uso e administração de medicamento. [Internet]. Brasília( DF); 2013[Acesso 12 mar 2019]. Disponível em: https://proqualis.net/sites/proqualis.net/files/000002490IQmwD8.pdf

8. Beaton D, Bombardier C, Guillemin F, Ferraz MB. Recommendations for the crosscultural adaptation of the DASH \& QuickDASH outcome measures. [Internet]. Institute for Work \& Health; 2007[cited 2019 mar 12]. Available from: http://www.dash. iwh.on.ca/sites/dash/files/downloads/cross_cultural_adaptation_2007.pdf .

9. Sandoval, L. J. S. Tradução, adaptação transcultural e validação do instrumento segurança do paciente na administração de medicamentos na pediatria. 2019. $137 \mathrm{f}$. Tese (Doutorado em Enfermagem) - Faculdade de Farmácia, Odontologia e Enfermagem, Universidade Federal do Ceará, Fortaleza, 2019.

10. Echevarría-Guanilo ME, Gonçalves N, Romanoski PJ. Psychometric properties of measurement instruments: conceptual basis and evaluation methods - part II. Texto contexto - enferm. 2019;28: e20170311. doi: 10.1590/1980-265x-tce-2017-0311

11. Pasquali L. Teoria dos testes na psicologia e na educação. 1 ed. Vozes, Petrópolis; 2017. 481 p.

12. Lobiondo-Wood G, Haber J. Nursing Research: methods and critical appraisal for evidence-based practice. 9 ed. St. Louis Missouri, Elsevier, 2018. 552 p.

13. De Souza MJ, Real DSS, Cunha ICKO, Bohomol E. Safe practices for medication administration: instrument construction and validation. Enferm. foco. 2017;8(4):20-25. doi: 10.21675/2357-707X.2017.v8.n4.973

14. Timm M, Rodrigues MCS. Cross-cultural adaptation of safety culture tool for Primary Health Care. Acta paul. enferm. 2016;29(1):26-37. doi: 10.1590/19820194201600005.

15. Gomes ALA, Joventino ES, Lima KF, Dodt RCM, Almeida PC, Ximenes LB. Validation and reliability of the scale self-efficacy and their child's level of asthma control. Rev. Bras. Enferm. 2018;71(2):406-12. doi: 10.1590/0034-7167-2016-0528.

16. Rogers JW, Fleming M, Tipton J, Ward A, Garey KW, Pitman EP. Investigating inpatient medication administration using the theory of planned behavior. Am J Health Syst Pharm. 2017;74(24):2065-70. doi: 10.2146/ajhp160502.

17. Petersen RS, Tennant A, Nakagawa TH, Marziale MHP. Translation, adaptation and validation of the nurse-work instability scale to brazilian portuguese. Rev. LatinoAm. Enfermagem. 2019;27:e3170. doi: 10.1590/1518-8345.2943.3170.

18. Pereira FMV, Lam SC, Gir E. Cultural adaptation and reliability of the compliance with standard precautions scale (CSPS) for nurses in Brazil. Rev. Latino-Am. Enfermagem. 2017; 25: e2850. doi: 10.1590/1518-8345.1204.2850.

19. Keszei AP, Novak M, Streiner DL. Introduction to health measurement scales. J Psychosom Res. 2010;68(4):319-23. doi: 10.1016/j.jpsychores.2010.01.006. 
